\title{
Dual-probe spectroscopic fingerprints of defects in graphene
}

\author{
Settnes, Mikkel; Power, Stephen; Petersen, Dirch Hjorth; Jauho, Antti-Pekka
}

Published in:

Physical Review B

Publication date:

2014

Document Version

Publisher's PDF, also known as Version of record

Link back to DTU Orbit

Citation (APA):

Settnes, M., Power, S., Petersen, D. H., \& Jauho, A-P. (2014). Dual-probe spectroscopic fingerprints of defects in graphene. Physical Review B, 90(3), 035440.

\section{General rights}

Copyright and moral rights for the publications made accessible in the public portal are retained by the authors and/or other copyright owners and it is a condition of accessing publications that users recognise and abide by the legal requirements associated with these rights.

- Users may download and print one copy of any publication from the public portal for the purpose of private study or research.

- You may not further distribute the material or use it for any profit-making activity or commercial gain

- You may freely distribute the URL identifying the publication in the public portal

If you believe that this document breaches copyright please contact us providing details, and we will remove access to the work immediately and investigate your claim. 


\title{
Dual-probe spectroscopic fingerprints of defects in graphene
}

\author{
Mikkel Settnes, ${ }^{*}$ Stephen R. Power, Dirch H. Petersen, and Antti-Pekka Jauho \\ Center for Nanostructured Graphene (CNG), Department of Micro and Nanotechnology, DTU Nanotech, \\ Technical University of Denmark, DK-2800 Kongens Lyngby, Denmark
}

(Received 2 May 2014; revised manuscript received 23 June 2014; published 24 July 2014)

\begin{abstract}
Recent advances in experimental techniques emphasize the usefulness of multiple scanning probe techniques when analyzing nanoscale samples. Here, we analyze theoretically dual-probe setups with probe separations in the nanometer range, i.e., in a regime where quantum coherence effects can be observed at low temperatures. In a dual-probe setup the electrons are injected at one probe and collected at the other. The measured conductance reflects the local transport properties on the nanoscale, thereby yielding information complementary to that obtained with a standard one-probe setup (the local density of states). In this work we develop a real-space Green's function method to compute the conductance. This requires an extension of the standard calculation schemes, which typically address a finite sample between the probes. In contrast, the developed method makes no assumption of the sample size (e.g., an extended graphene sheet). Applying this method, we study the transport anisotropies in pristine graphene sheets, and analyze the spectroscopic fingerprints arising from quantum interference around single-site defects, such as vacancies and adatoms. Furthermore, we demonstrate that the dual-probe setup is a useful tool for characterizing the electronic transport properties of extended defects or designed nanostructures. In particular, we show that nanoscale perforations, or antidots, in a graphene sheet display Fano-type resonances with a strong dependence on the edge geometry of the perforation.
\end{abstract}

DOI: 10.1103/PhysRevB.90.035440 PACS number(s): 68.37.-d, 73.22.Pr, 72.10.Fk, 73.63.-b

\section{INTRODUCTION}

A key step towards developing novel applications for graphene and other two-dimensional materials [1-3] is to obtain a detailed understanding of their electron transport properties on the nanoscale [4]. At these length scales structural details play a crucial role due to the restricted dimensionality. Thus, studying spatially resolved electron transport becomes important, especially near defects and boundaries, which dramatically affect the conductance of a device $[5,6]$.

Scanning tunneling microscopy (STM) $[7,8]$ is an important noninvasive method for studying the electronic structure of surfaces. Nanometer-scale STM measurements, yielding both local density of states (LDOS) and topographic details, are extensively used both theoretically [9-16] and experimentally [17-22] in the study of graphene. However, the STM measures only local properties, whereas we often want to obtain information about the transport properties for electrons traversing the sample. On the other hand, transport properties are most commonly measured by using invasive macroscopic contacts. Such contacts represent only a minor perturbation in large systems, but can be the main source of scattering in nanoscale devices. Here we evaluate the conductance between two STM-like tips, i.e., a situation where nanoscale transport properties can be extracted with noninvasive probes. The considered regime is thus between the single-STM setup and the fixed macroscopic contacts.

The envisaged technique requires independently positioned point probes to act as input and output. Such setups have been achieved experimentally [23-28] and the recent progress is reviewed in detail in Refs. [29,30]. State-of-the-art experimental techniques [24,31] allow for tip separations down to $50-100 \mathrm{~nm}$. Compared to the conventional single-STM

*mikse@ nanotech.dtu.dk setups, which reflect local properties, the multiprobe signal provides additional information being a transport quantity. Multiprobe measurements have been used to characterize several systems: anisotropic transport [32], nanowires [24,33], carbon nanotubes [34], graphene nanoribbons [31], grain boundaries both in graphene [35,36] and other materials [37], and monolayer and bilayer graphene [38-40].

Graphene-based materials are particularly interesting because the mean-free path in high-quality samples is comparable to or perhaps even longer than the probe separation [31]. As a result, the dual-STM setup is effectively in the phase-coherent regime at low temperatures. In this regime, structural details, such as single-site scattering centers, edges, or grain boundaries, limit the conductance, such that quantum interference phenomena become visible in the transmission between the probes. This gives information about how the electrons travel through the sample and is not limited to local properties as is the single-STM measurement.

In a recent work [41], the present authors proposed a dual-probe setup keeping one fixed probe while the other probe operates in the scanning mode. We used real-space conductance maps to explore quantum interference effects near defects and edges in graphene. Fourier transforms of the real-space conductance maps allowed us to extract further details, and in particular they revealed information about intra- and intervalley scattering due to these defects. In the present work, we extend the theoretical investigation to the spectroscopic mode of the dual-probe system, where two fixed probes operate in the presence of an applied gate, which allows the Fermi energy to be varied. While we focus on graphene as an illustrative example, particularly suited for the observation of quantum interference phenomena, the methodology is general and applicable to other surfaces or two-dimensional materials. We use a combination of numerical calculations and analytic expressions to explain the spectroscopic fingerprints observed both in pristine graphene and in the presence of 
vacancies and adatoms. Finally, we extend the framework to nanostructures such as perforations.

The paper is organized as follows: Section II introduces the real-space Green's function (GF) method and highlights the conceptual differences compared to standard recursive GF techniques. Section III considers dual-probe spectroscopy of pristine graphene based on both analytical approximations of the GF and numerical calculations. Section IV introduces defects where both single vacancies and adatoms are considered in the high-symmetry directions, but also randomly placed in the sample. Finally, Sec. V considers perforations of the graphene lattice with different edge geometries.

\section{METHOD}

\section{A. Transport calculations using point probes}

The transport setup consists of a device region and two leads as illustrated in Fig. 1. We describe the leads by the surface $\mathrm{GF}, g_{S}$, which couples to the device region that is described by the retarded/advanced GF $\boldsymbol{G} / \boldsymbol{G}^{\dagger}$. We view $g_{S}$ as a known quantity (a simple analytic model is used below, but more elaborate models are readily incorporated in the formalism), and solve $\boldsymbol{G} / \boldsymbol{G}^{\dagger}$ from the appropriate Dyson equation; see below. The main difference between the setup sketched in Fig. 1 and the standard Landauer setup [42], where left and right lead couple to the edge of a finite device region, is that the device region is now infinite. Standard recursive methods, treating infinite systems, use periodic boundary conditions. However, imposing periodic boundary conditions for the twopoint probe setup would lead to a spurious repetition of the probes. As a consequence we require a real-space formalism ensuring that the probes only appear locally.

\section{B. Real-space graphene Green's function}

The basic building block of our method is the real-space representation of the GF for an infinite pristine graphene sheet, $\boldsymbol{G}^{0}$. This object is computed using a nearest-neighbor tight-

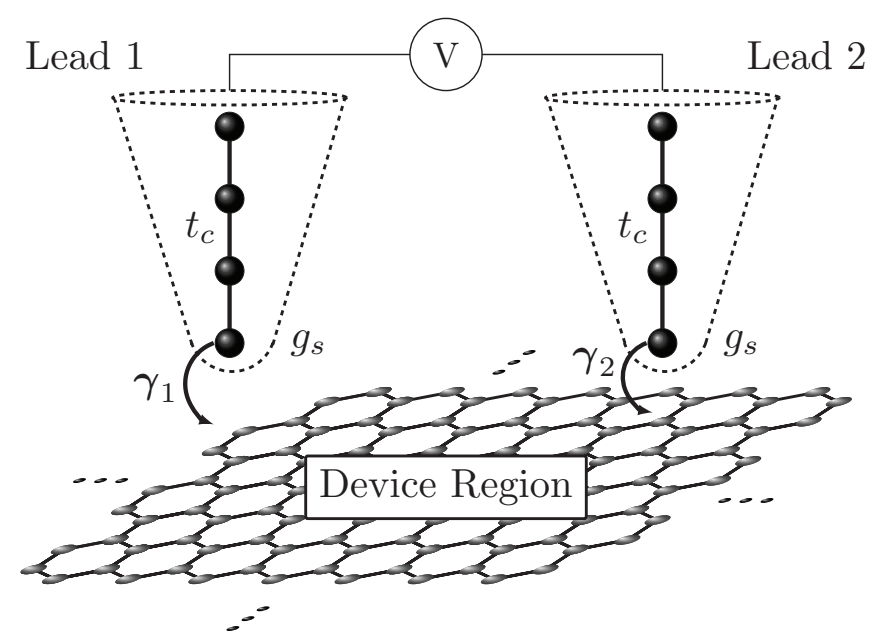

FIG. 1. Setup sketch including the leads modeled as onedimensional chains with a hopping $t_{c}$ between the sites. The surface Green's functions $g_{s}$ are indicated together with the coupling $\gamma^{1 / 2}$ between the lead and the graphene sample. binding model, and the GF element connecting sites $i$ and $j$ is given by

$$
G_{i j}^{0}(z)=\frac{1}{\Omega_{\mathrm{BZ}}} \int d^{2} \boldsymbol{k} \frac{N_{i j}(z) e^{i \boldsymbol{k} \cdot\left(\boldsymbol{r}_{j}-\boldsymbol{r}_{i}\right)}}{z^{2}-\gamma_{c c}^{2}|f(\boldsymbol{k})|^{2}},
$$

where $z=E+i 0^{+}$is the energy, $\Omega_{\mathrm{BZ}}$ is the area of the first Brillouin zone, and the carbon-carbon hopping integral is $\gamma_{c c} \approx-2.7 \mathrm{eV}$ [43]. The position of site $i$ is denoted by $\boldsymbol{r}_{i}=$ $m_{i} \boldsymbol{a}_{1}+n_{i} \boldsymbol{a}_{2}$ in units of the lattice vectors $\boldsymbol{a}_{1}$ and $\boldsymbol{a}_{2}$ with $m_{i}$ and $n_{i}$ being integers. We introduced the definition $N_{i j}(z)=z$, when $i$ and $j$ are on the same sublattice and $N_{i j}(z)=\gamma_{c c} f(\boldsymbol{k})$ if $i$ and $j$ are on opposite sublattices $\left(N_{j i}=N_{i j}^{*}\right)$. Finally, we use the definition $f(\boldsymbol{k})=1+e^{i \boldsymbol{k} \cdot \boldsymbol{a}_{1}}+e^{i \boldsymbol{k} \cdot \boldsymbol{a}_{2}}$.

The two-dimensional integral Eq. (1) can be converted to a single integration using complex contour techniques [44]. The remaining integration can be treated using standard numerical integration. This approach is valid regardless of the separation between the points $i$ and $j$; even long distance terms are easily obtained with only a minor complications arising in the convergence of Eq. (1) due to the rapidly oscillating phase.

\section{Including defects}

To include modifications to the pristine lattice, we use the Dyson equation:

$$
\boldsymbol{G}=\boldsymbol{G}^{0}+\boldsymbol{G}^{0} \boldsymbol{V} \boldsymbol{G}=\left(\mathbf{1}-\boldsymbol{G}^{0} \boldsymbol{V}\right)^{-1} \boldsymbol{G}^{0},
$$

where $\boldsymbol{G}^{0}$ is the pristine GF matrix and $\boldsymbol{V}$ is the perturbation. Any local perturbation (e.g., adatoms, vacancies, coupling to leads) can be included using this technique. Accurate parametrizations for many of these perturbations can be obtained using density functional theory $[45,46]$.

We note that the dimension of the $\boldsymbol{V}$ matrix is determined by the number of the modified sites. Thus, for $N$ modified sites one needs to solve a $N \times N$ system, and the computational cost thus follows the number of defect and contact sites, rather than the sample size which is usual for recursive GF methods.

All perturbations to the pristine lattice are added in real space using Eqs. (1) and (2), as opposed to describing them with additional terms in the reciprocal space Hamiltonians. This ensures that modifications are added locally and not repeated via periodic boundary conditions. The approach is well suited to situations where the majority of the sample is pristine, as unmodified graphene is computationally "free."

\section{Transmission}

The transmission coefficient between the two probes, $\mathcal{T}_{12}$, yields the zero-temperature conductance as $\mathcal{G}_{12}=\frac{2 \mathrm{e}^{2}}{\mathrm{~h}} \mathcal{T}_{12}$ (here we treat the spin-degenerate case). The transmission is given by $[42,47]$

$$
\mathcal{T}_{12}(E)=\operatorname{Tr}\left[\boldsymbol{G}(E) \boldsymbol{\Gamma}_{1}(E) \boldsymbol{G}^{\dagger}(E) \boldsymbol{\Gamma}_{2}(E)\right],
$$

where $E$ is the energy, $\boldsymbol{G}$ is the full Green's function Eq. (2) (including the sites coupling to the leads), and $\boldsymbol{\Gamma}_{1 / 2}$ is the coupling to the leads given as $\boldsymbol{\Gamma}_{1 / 2}(E)=i\left(\boldsymbol{\Sigma}_{1 / 2}-\boldsymbol{\Sigma}_{1 / 2}^{\dagger}\right)$. The self-energies $\Sigma_{1 / 2}$ of the leads are calculated from the coupling matrix between the lead and the sample $\boldsymbol{\gamma}_{1 / 2}$ and the surface GF of the lead $g_{s}$; i.e., $\boldsymbol{\Sigma}_{1 / 2}=\boldsymbol{\gamma}_{1 / 2}^{\dagger} g_{s} \boldsymbol{\gamma}_{1 / 2}$. We use a linear 
atomic chain model for the leads where the surface GF is known exactly [48]: $g_{S}=\frac{E \pm \sqrt{E^{2}-4 \gamma_{l}^{2}}}{2 \gamma_{l}^{2}}$, where $\gamma_{l}$ is the coupling between the sites in the linear chain (here $\gamma_{l}=\gamma_{c c}$ is used). The parameters are chosen to ensure a constant DOS in the leads in the considered energy interval.

The coupling between the graphene and the tip of the probes is calculated using the Tersoff-Hamann approach $[49,50]$ :

$$
\gamma_{j}=\gamma_{0} w_{j} e^{-d_{j} / \lambda} \cos \left(\theta_{j}\right),
$$

where $\theta_{j}$ and $d_{j}$ are the angle and the distance, respectively, between the tip apex and site $j$, and $w_{j}=e^{-a d_{j}^{2}} / \sum_{m} e^{-a d_{m}^{2}}$, $\lambda=0.85 \AA$, and $a=0.6 \AA^{-2}$ are constants chosen in accordance to Refs. [50,51]. $\gamma_{0}$ is a scaling factor, which in practical calculations is set to $\gamma_{0}=10 \gamma_{c c}$.

When considering a probe coupling to a single site, the transmission in Eq. (3) reduces to the following simple form:

$$
\mathcal{T}_{12}(E)=\left(2 \pi \gamma_{1} \gamma_{2} \rho_{\text {lead }}\right)^{2}\left|G_{12}(E)\right|^{2},
$$

where $\rho_{\text {lead }}=-\operatorname{Im}\left(g_{s}\right) / \pi$ is the constant density of states of the last atom of lead. Hence the only energy dependence originates from the GF term.

From Eq. (5) we notice that the transmission scales with the DOS of the leads. The transmission also scales with the coupling to the probes as $\sim \gamma_{1}^{2} \gamma_{2}^{2}$. As $\gamma_{1 / 2}$ depend exponentially on the distance between the tip and the sample, this means that decreasing the distance between sample and tip by $\sim 1 \AA$ increases the coupling which in turn increases the transmission by a factor of $\sim 100$.

In what follows, we consider STM-like probes (i.e., probes which couple only to a very limited number of sites in the sample) in order to obtain transparent results giving insight into the processes which dominate the transport between the point probes. More realistic or larger probes may be included within the presented framework by increasing the number of graphene lattice sites that couple to the probes or by substituting the semi-infinite monoatomic chain by other surface GFs.

Finally, it is noted that we consider the low-temperature and low-bias regime and therefore ignore inelastic effects such as phonon scattering [52]. Here we also neglect the possible nonplanarity of the graphene sheet, either due to the intrinsic ripples [53] or caused by one of the probes [38]. However, we previously discussed the effect of ripples on dual-probe scanning mode calculations in Ref. [41].

\section{PRISTINE GRAPHENE}

We first consider the case of pristine graphene without defects. In this case we can gain a transparent understanding by the so-called stationary phase approximation (SPA) [44] to the GF in Eq. (1). The SPA is valid for the high-symmetry directions (armchair or zigzag) and for separations between the $i$ and $j$ sites exceeding a few lattice spacings. Using the SPA, the graphene GF in Eq. (1) can be expressed as

$$
\begin{aligned}
G_{i j, \mathrm{SPA}}^{0, a c} & =\frac{\mathcal{A}(E) e^{i \mathcal{Q}(E) d_{i j}}}{\sqrt{d_{i j}}}, \\
G_{i j, \mathrm{SPA}}^{0, z z} & =\sum_{\eta= \pm} \frac{\mathcal{A}^{\eta}(E) e^{i \mathcal{Q}^{\eta}(E) d_{i j}}}{\sqrt{d_{i j}}},
\end{aligned}
$$

where $\mathcal{A}(E)$ is an energy-dependent amplitude and $\mathcal{Q}(E)$ is the Fermi wave vector in the armchair and zigzag directions. The coefficients are given in the Appendix and derived in Ref. [44].

Inserting Eq. (6) into Eq. (5) gives the distance dependence of the transmission, $\mathcal{T}_{12} \propto 1 / d_{12}$. Consequently the resistance scales linearly with probe separation, $R \propto d_{12}$.

Consider now the case when the separation between the two probes is in the armchair direction. Using Eq. (6a), we find that the transmission coefficient increases linearly with energy. The linear increase of $\mathcal{T}^{(a c)} \propto|\mathcal{A}|^{2} / d_{12}$ originates from the fact that $|\mathcal{A}|^{2}$ grows linearly with energy for low energies; see the Appendix.

The zigzag direction is more complicated because of the two terms in Eq. (6b), caused by the two nonidentical sides of the Fermi surface along the zigzag direction:

$$
\begin{aligned}
\mathcal{T}_{12}^{(z z)} \times d_{12} \propto & \left|\sum_{\eta= \pm} \mathcal{A}^{\eta} e^{i \mathcal{Q}^{\eta} d_{12}}\right|^{2}=\left|\mathcal{A}^{+}\right|^{2}+\left|\mathcal{A}^{-}\right|^{2} \\
& +\left|\mathcal{A}^{+}\right|\left|\mathcal{A}^{-}\right| \cos \left(\left[\mathcal{Q}^{+}-\mathcal{Q}^{-}\right] d_{12}\right) .
\end{aligned}
$$

In addition to the linear increase (the first two terms), we also find an oscillating term. The oscillation period decreases with increasing energy due to the energy dependence of $\mathcal{Q}^{+}-\mathcal{Q}^{-}$. We therefore expect a more rapid oscillation for higher values of the Fermi energy.

In Figs. 2(b) and 2(c) we plot the energy-dependent transmission for $d_{i j}$ parallel to either armchair (b) or zigzag (c) for probe separation $\sim 50 \mathrm{~nm}$. The transmissions are calculated using both Eq. (6) (dots) and using a numerical evaluation of Eq. (1) (line). We note an almost perfect match for all energies, which confirms the validity of the SPA approach.

In Fig. 2(d) we consider a direction rotated $\theta \approx 11^{\circ}$ relative to the armchair direction. Consequently the oscillation period depends on the rotation angle $\theta$, as defined $n$ Fig. 2(a). The oscillation is a consequence of the asymmetry of the Fermi surface in the given direction and is therefore a fingerprint of the crystalline direction between the probes.

The GFs for all other separations (except armchair) have the same form as Eq. (6b) [44]. So the transmission generally takes a form equivalent to Eq. (7) but with different expressions for $\mathcal{Q}^{+}$and $\mathcal{Q}^{-}$, which depend on the direction of separation. In the limit of low energies we can expand the coefficients as $|\mathcal{A}|^{2} \propto E$ and $\left(\mathcal{Q}^{+}-\mathcal{Q}^{-}\right) \propto E^{2} / \nu(\theta)$. Here $\nu(\theta)$ is an oscillation period that depends on the angle $\theta$ [defined in Fig. 2(a)]. Accordingly $\theta=0$ denotes armchair separation and $\theta=30^{\circ}$ denotes zigzag separation between the probes. The energy dependence of the transmission in Eq. (7) now becomes

$$
\mathcal{T}_{12} \propto E \cos \left[E^{2} d_{12} / v(\theta)+\phi_{p h}\right],
$$

where $\phi_{p h}$ is a phase factor, which is independent of the direction but depends on the distance and the exact atoms coupling to the probes. If we plot $\mathcal{T}_{12} / E$ as a function of $d_{12} E^{2}$ we can determine the period $v(\theta)$ as the lowest full period of oscillation in the $\mathcal{T}_{12} / E$ vs $d_{12} E^{2}$ plot for the corresponding angle $\theta$. In Fig. 2(e), we plot $v(\theta)$ as a function of angle. Figure 2(e) is the average of many individual calculations of $\nu(\theta)$ for separations ranging from 20 to $100 \mathrm{~nm}$.

From Fig. 2(e) we conclude that $v(\theta)$ provides a fingerprint of the probe separation direction. Furthermore $v(\theta)$ enables 
(a)
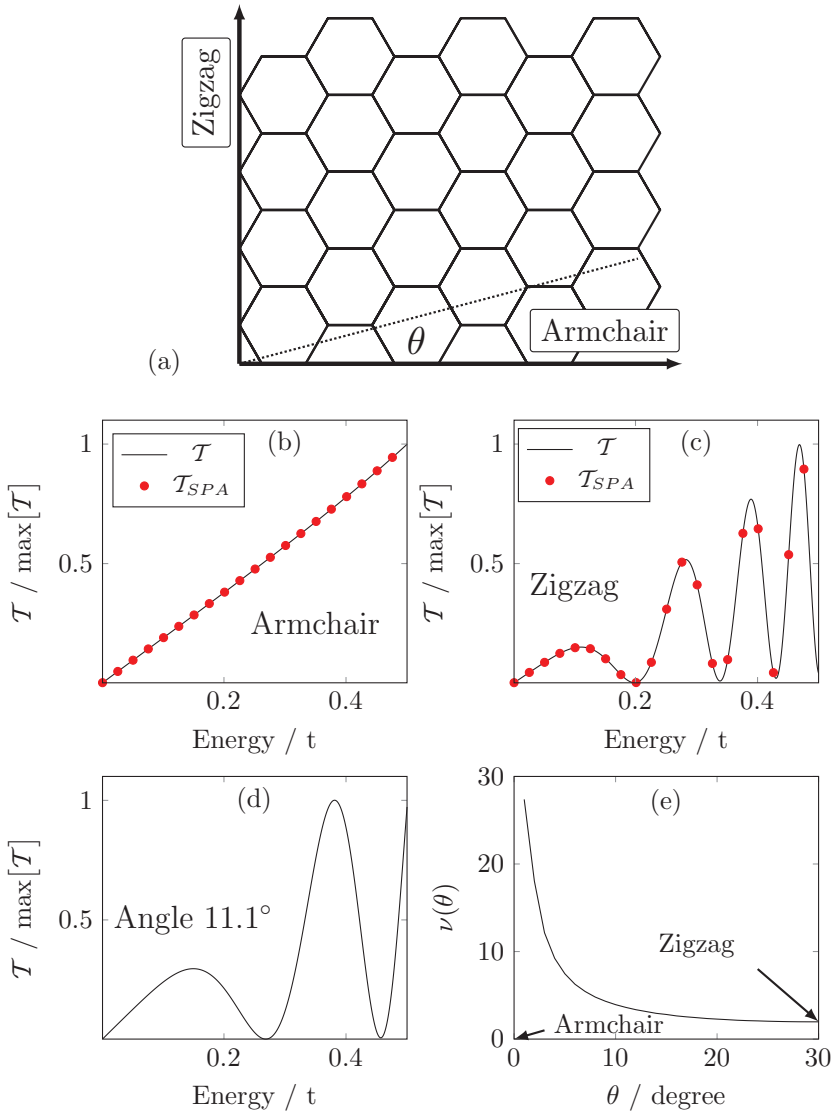

FIG. 2. (Color online) (a) Sketch showing the pristine sample and the rotation angle $\theta$ from the armchair direction. (b)-(d) The transmission as a function of energy between the two leads separated by $50 \mathrm{~nm}$ along (b) armchair, (c) zigzag, and (d) rotated $\theta=11.1^{\circ}$ from the armchair direction. In (b) and (c) the transmission calculated using the SPA is indicated (red dots). (e) The oscillation period $v(\theta)$ (see main text for definition) is plotted against rotation angle $\theta$ as defined in (a). The curve is constructed by averaging over many individual calculations with distances ranging from 20 to $100 \mathrm{~nm}$.

us to determine the crystalline direction with a simple spectroscopic measurement provided we know the distance between the probes and that the gate is kept sufficiently small.

\section{SIMPLE DEFECTS}

Next we consider defects such as vacancies and adatoms. To obtain an analytical treatment in this case let the defects be coupled to a group of sites denoted 0 and the probes coupled to sites denoted 1 and 2. We restate the Dyson equation [Eq. (2)] using the $t$-matrix formalism

$$
\boldsymbol{G}_{12}=\boldsymbol{G}_{12}^{0}+\boldsymbol{G}_{10}^{0} \mathbf{t}_{00} \boldsymbol{G}_{02}^{0}
$$

where

$$
\mathbf{t}_{00}=\left(\mathbf{1}-\boldsymbol{V}_{00} \boldsymbol{G}_{00}^{0}\right)^{-1} \boldsymbol{V}_{00} .
$$

Inserting this into Eq. (3) we obtain

$$
\begin{aligned}
\mathcal{T}_{12} \propto & \operatorname{Tr}\left[\left(\boldsymbol{G}_{12}+\boldsymbol{G}_{10} \mathbf{t}_{00} \boldsymbol{G}_{02}\right)\left(\boldsymbol{G}_{12}^{\dagger}+\boldsymbol{G}_{02}^{\dagger} \mathbf{t}_{00}^{\dagger} \boldsymbol{G}_{10}^{\dagger}\right)\right] \\
= & \operatorname{Tr}\left[\boldsymbol{G}_{12} \boldsymbol{G}_{12}^{\dagger}+\left(\boldsymbol{G}_{10} \mathbf{t}_{00} \boldsymbol{G}_{02}\right)\left(\boldsymbol{G}_{10} \mathbf{t}_{00} \boldsymbol{G}_{02}\right)^{\dagger}\right. \\
& \left.+2 \operatorname{Re}\left\{\left(\boldsymbol{G}_{10} \mathbf{t}_{00} \boldsymbol{G}_{02}\right) \boldsymbol{G}_{12}^{\dagger}\right\}\right] .
\end{aligned}
$$

Equation (11) is generally applicable. If the probes and the defect couple to single sites all matrices reduce to scalar quantities and enable simple analytic expressions. For example, we use the SPA expression Eq. (6a) when both probes and defects are along the armchair direction

$$
\begin{aligned}
\Delta \mathcal{T}_{12}= & \mathcal{T}_{12}-\mathcal{T}_{12}^{0} \propto \frac{|\mathcal{A}|^{4}}{d_{10} d_{20}}\left|t_{00}\right|^{2} \\
& -\frac{|\mathcal{A}|^{3}}{\sqrt{d_{10} d_{20} d_{12}}} \operatorname{Re}\left\{(1+i) t_{00} e^{i \mathcal{Q}\left(d_{10}+d_{20}-d_{12}\right)}\right\},
\end{aligned}
$$

where $\mathcal{T}_{12}^{0}$ is the pristine transmission, $d_{12}$ denotes the distance between the two probes, and $d_{10}$ and $d_{20}$ denote the distance between the defect site and probes 1 and 2, respectively.

Assuming the defect lies between the probes, i.e., $d_{12}=d_{10}+d_{20}$, we get from Eq. (12)

$$
\Delta \mathcal{T}_{12} \propto \frac{|\mathcal{A}|^{4}}{d_{10} d_{20}}\left|t_{00}\right|^{2}-\frac{|\mathcal{A}|^{3}}{\sqrt{d_{10} d_{20} d_{12}}} \operatorname{Re}\left\{(1+i) t_{00}\right\} .
$$

Thus, a change in transmission occurs due to the backscattering at the defect. This was also observed in Ref. [41] where one probe scanned around the defect position to obtain a real-space image of the transmission change. The size and form of $\Delta \mathcal{T}_{12}$ depend on the type of defect through $t_{00}$.

For the defect on either side of the probes, i.e., $d_{10}=d_{12}+$ $d_{20}$, Eq. (12) becomes

$$
\Delta \mathcal{T}_{12} \propto \frac{|\mathcal{A}|^{4}}{d_{10} d_{20}}\left|t_{00}\right|^{2}-\frac{|\mathcal{A}|^{3}}{\sqrt{d_{10} d_{20} d_{12}}} \operatorname{Re}\left\{(1+i) t_{00} e^{2 i \mathcal{Q} d_{20}}\right\} .
$$

The result for the impurity on the other side of the probes $\left(d_{20}=d_{12}+d_{10}\right)$ is obtained by interchanging 1 and 2 . The case in Eq. (14) gives rise to oscillations as we change the energy (by changing $\mathcal{Q}$ ). The oscillations are a consequence of quantum interference between the outgoing wave from the output probe and the scattered wave. Similar expressions as Eqs. (13) and (14) can be derived for the zigzag separation, but the simple form is complicated by the two interfering terms in Eq. (6b).

Equations (13) and (14) show that the effect of the impurity enters through the $t$ matrix, which depends on the type of impurity. In this section we consider two specific defects: vacancies and adatoms. Vacancies are modeled as a change of the on-site energy, $V_{00} \rightarrow \infty$. On the other hand, adatoms are modeled with an energy-dependent self-energy $\Sigma^{\alpha}$, describing a resonant level with energy $\epsilon_{\alpha}$, coupled to the graphene sample with coupling constant $\gamma_{\alpha}$; i.e., $V_{00}=\Sigma_{00}^{\alpha}=\left|\gamma_{\alpha}\right|^{2} /$ $\left(E+i 0^{+}-\epsilon_{\alpha}\right)$. The $t$ matrices become $[54,55]$

$$
\text { Vacancy: } \begin{aligned}
t_{00} & =\frac{V_{00}}{1-V_{00} G_{00}^{0}} \rightarrow-\frac{1}{G_{00}^{0}}, \\
\text { Adatom: } t_{00} & =\frac{\Sigma_{00}^{\alpha}}{1-\Sigma_{00}^{\alpha} G_{00}^{0}}=\left(\Sigma_{00}^{\alpha-1}-G_{00}^{0}\right)^{-1} \\
& =\frac{\left|t_{\alpha}\right|^{2}}{E-\epsilon_{\alpha}-\left|t_{\alpha}\right|^{2} G_{00}^{0}} .
\end{aligned}
$$

The adatom gives rise to a resonant level whose position is determined by both $\epsilon_{\alpha}$ and $\gamma_{\alpha}$. We choose parameters from 


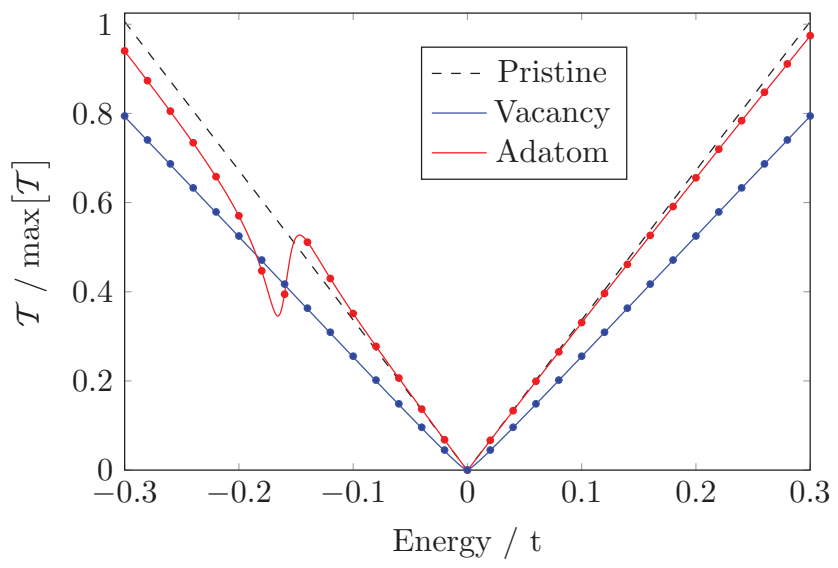

FIG. 3. (Color online) The transmission as a function of energy for pristine graphene (dashed), vacancy (red), and adatom (blue). The impurity is in between probes, which are separated by $\sim 50 \mathrm{~nm}$ along the armchair direction. The dots denote a similar calculation using the SPA expression Eq. (13). The parameters for the adatom are chosen as in Ref. [56] as $\epsilon_{\alpha}=-0.185|t|$ and $t_{\alpha}=0.37|t|$.

Ref. [56] as $\epsilon_{\alpha}=-0.185|t|$ and $t_{\alpha}=0.37|t|$. This gives a resonant level within the energy interval of consideration.

Figure 3 shows the numerical result compared to the analytical expression Eq. (13) for both a vacancy and an adatom. The impurities are located equidistant $\left(d_{10}=d_{20}=d_{12} / 2\right)$ from the two probes. Again, we observe an almost perfect match between the analytic (symbols) and numerical (lines) results. The vacancy gives rise to an overall reduction in transmission due to scattering, while the adatom leads to a smaller reduction of transmission, except at the resonance. Especially at resonance the level of the adatom interacts strongly with the continuum of the graphene states giving rise to the asymmetric Fano-type resonance [57] observed at approximately $-0.15 \mathrm{eV}$ in Fig. 3. Similar results are obtained for the zigzag direction, but superimposed onto the characteristic zigzag oscillation discussed in Sec. III.

\section{A. Impurity positions}

To investigate the influence of adatom position on the resonance, we now move the adatom away from the highsymmetry point between the probes. First, the adatom is moved along the line connecting the probes such that it is no longer equidistant from the probes. These positions are shown by the red and green squares in Fig. 4(a). The corresponding dual-probe transmissions are shown in Fig. 4(b) and the change relative to the pristine graphene sheet is shown in Fig. 4(c). Furthermore, both panels include the transmission for the equidistant impurity (blue curve) for comparison. Likewise Figs. 4(d) and 4(e) show the corresponding transmissions as the adatom is moved perpendicular to the line separating the probes while keeping the impurity equidistant to the probes.

First, we consider the parallel case. Here the adatom is either in between the probes, yet closer to one of them [green square in Fig. 4(a)], or to the far side of one of the probes [red square in Fig. 4(a)]. The Fano-type resonance is present for both positions and only the form changes. However, (a)
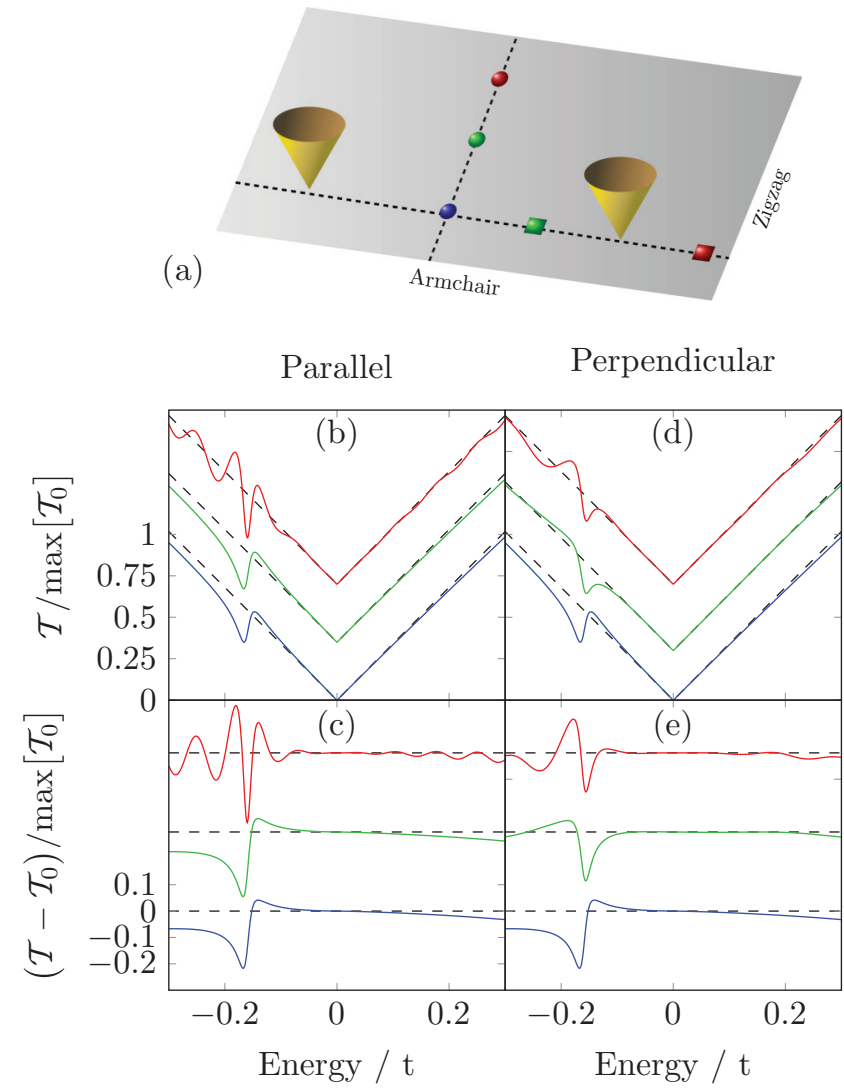

FIG. 4. (Color online) (a) Sketch illustrating the two probes separated along the armchair direction by $\sim 50 \mathrm{~nm}$. The marks refers to impurity positions. Blue is along the line of separation and equidistant of the probes. The green and red squares are moved relative to the blue site along the armchair direction (parallel) by $12.8 \mathrm{~nm}$ and $34 \mathrm{~nm}$, respectively. The transmission for the parallel translation is shown in (b) and (c). The green and red circles are equidistant of the probes but moved along the zigzag direction (perpendicular) to $7.4 \mathrm{~nm}$ and $17.2 \mathrm{~nm}$, respectively. The transmission function for impurities in these positions is shown in (d) and (e). The zero point for the curves has been translated for better distinction between curves.

when the impurity does not lie between the probes (red square), additional oscillations arise. This can be understood by comparing Eqs. (13) and (14) where the difference is the term $\exp \left(2 i \mathcal{Q} d_{20}\right)$. This term gives rise to oscillations through the energy dependence of $\mathcal{Q}$. The oscillations have the same origin as these investigated in real space in Ref. [41] while scanning one probe around the impurity. We notice the same effect for vacancy positions everywhere outside the high-symmetry positions.

The same type of oscillations are present for the perpendicular direction. In this case we have to consider the interference between the emitted wave and the scattered wave returning from the impurity in the direction of the second probe.

\section{B. Configurational average}

In an experimental setup, however, individual defects or adatoms can be difficult to locate. This makes investigations 


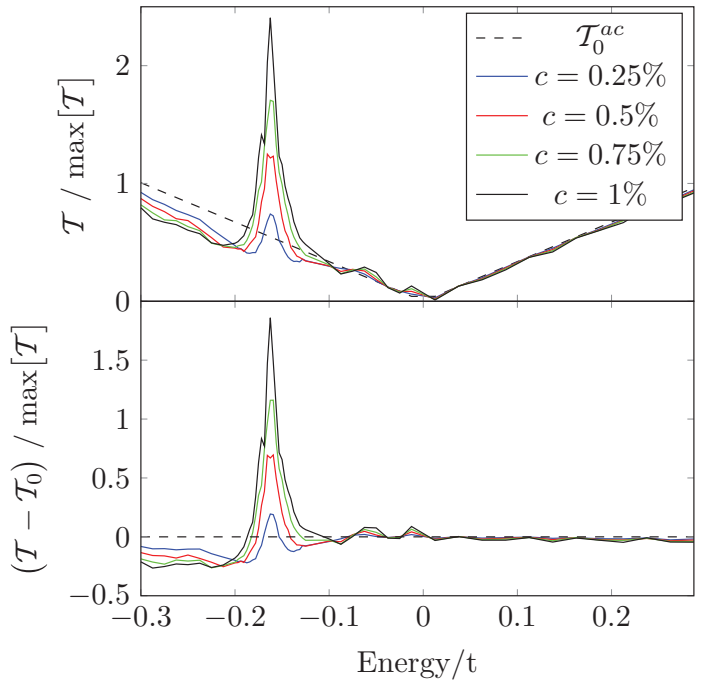

FIG. 5. (Color online) (a) Configuration-averaged transmission as a function of energy. (b) The difference between the averaged transmission and the pristine transmission. We place impurities in a $50 \times 85 \mathrm{~nm}$ square around the probes The unequal sides are chosen to take into account the probe separation direction. The curves are made from averaging $2 \times 10^{4}$ configurations.

of many randomly scattered defects important. We fix the two probes with an armchair separation of $50 \mathrm{~nm}$ and place adatoms randomly with varying concentration. The averaged transmissions are shown in Fig. 5. The transmission is almost unchanged at energies away from the resonance, despite the oscillations caused by individual impurity positions shown in Fig. 4. This shows that the oscillations, induced by interference between incoming and scattered waves, tend to average out for many defects. However, the resonance feature survives configurational averaging as is evident from Fig. 5. The signal is enhanced on resonance and an overall Fano-type resonance is present in Fig. 5(b) with a height that scales with impurity concentration. This suggests that the dual-probe setup can detect the type (position of resonant level) and concentration (peak height) of adatoms on the surface of a graphene sample without the need of scanning the exact position of the impurity as required for a single-probe measurement. This is in line with the suggested applications of graphene as a gas sensor [58,59]. In the case of random vacancies we see an overall decrease in the transmission following the impurity concentration. In this case a zero-energy peak is present due to localization effects around vacancies. This feature has been described in several works addressing the LDOS $[5,11,15]$.

\section{PERFORATED GRAPHENE}

Many applications require deliberate nanostructuring of the graphene in order to engineer its electronic structure. Therefore tools to investigate the transport properties of individual nanostructures are important in order to confirm the fabricated structure and its influence on nanoscale electron transport.

In this section we consider perforations in the pristine graphene sheet-the so-called antidots (see Fig. 6) [60].

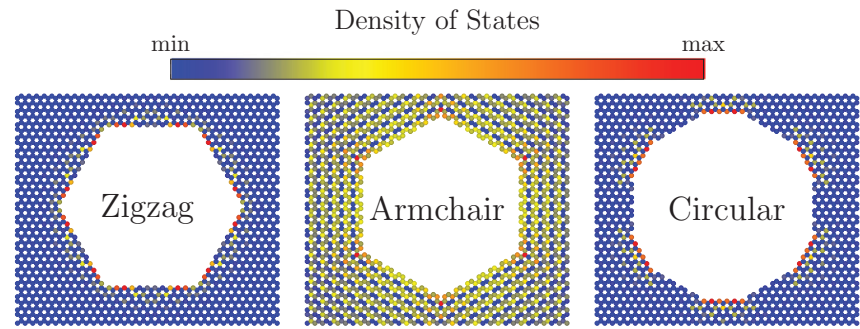

FIG. 6. (Color online) The density of states for $E=0.028|t|$ around antidots with different edge structures as indicated. The maps are individually scaled.

Several studies [61-63] show that arrays of antidots can induce a band gap in graphene. The effect of antidots on the electronic properties of graphene strongly depends on the exact edge geometry of the antidots. Therefore it is important to study the formation of single antidots and determine their edge configuration.

The perforations are modeled by removing the hopping matrix elements between sites around the edge of the hole, effectively disconnecting the sites from the rest of the graphene lattice.

We consider three possible edge geometries for antidots: zigzag, armchair, or circular; the last contains an alternating sequence of armchair and zigzag edges (Fig. 6). We calculate the transmission for each antidot type placed between probes separated in the armchair direction. The result is shown in Fig. 7(a). As expected the transmission is generally lowered by introduction of the perforation. A notable difference between the antidot types is a transmission dip present for the circular, and particularly zigzag, type antidots. This dip resembles the Fano-type resonance observed for single adatoms in Fig. 3. Figure 7(a) suggests that the resonant feature is connected to the zigzag edges, as the circular antidot consists of a mixture of zigzag and armchair edges. We therefore map the local density of states on sites around the antidot at the energy of the transmission dip (cf. Fig. 6). The DOS is localized around the zigzag edges as discussed in Ref. [63]. The localized zigzag edge states, being essentially dispersionless, resemble a single level and therefore create a Fano-type resonance in the transmission for antidots possessing zigzag edges. In addition, we notice the difference between the resonance of the circular and zigzag antidot on Fig. 7(a). The resonance of the pure zigzag edge has a sharper feature than the mixed edge (circular antidot). This leads to the conclusion that the resonance features can be related to the amount of zigzag edge present. Calculations performed with antidots of varying size (not shown) yield qualitatively the same result, but the position of the dip feature changes depending on the length of the zigzag edge present. This in turn can be used as a fingerprint to determine the edge profile of antidots and other nanostructures.

In Fig. 7(b) the robustness of the signal against edge disorder is investigated. We add an on-site potential, chosen randomly within $[-W, W]$, to the two rows of atoms around the antidot. Figure 7(b) shows the transmission for different disorder strengths averaged over several configurations. For weak edge disorder the resonance feature persists whereas it 

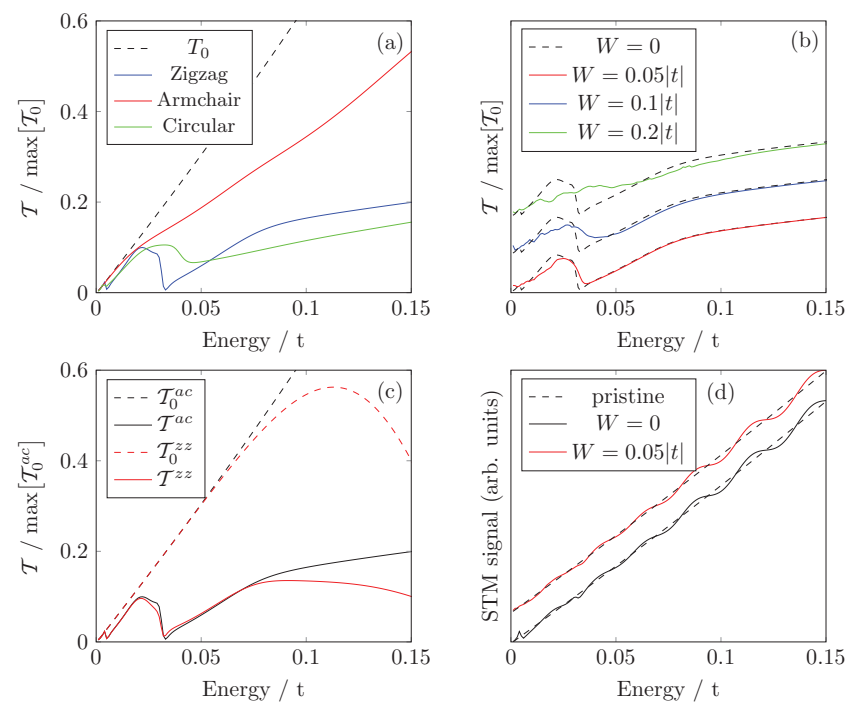

FIG. 7. (Color online) (a) The transmission for probes separated along the armchair direction $(\sim 50 \mathrm{~nm})$ for zigzag, armchair, and circular antidots, respectively. The antidot structures are shown in Fig. 6. (b) Transmission for the same zigzag antidot as (a) including disorder of varying strength. Each curve is an average of 50 different configurations and has been shifted relative to the others. (c) The transmission for the same zigzag antidot as (a), with probe separations $(\sim 50 \mathrm{~nm})$ along armchair and zigzag directions, respectively. (d) Single-probe spectroscopy of zigzag antidot with the same probe position as (a). Calculation both with and without disorder is included. The curves have been shifted relative to each other.

vanishes for higher disorder strengths as expected because high disorder tends to destroy the localized edge state giving rise to the resonance in the first place.

Next we consider the separation direction between the probes. The exact direction between the probes should not have a great impact on the spectroscopic fingerprints, if the dualprobe setup is to be a useful tool for characterization of larger nanostructures, such as antidots. Therefore we compare the transmission for both zigzag and armchair probe separations in Fig. 7(c) and note very similar behavior at low energies. We considered several nonsymmetric positions (not shown) all exhibiting the resonant feature in the same position. This shows that the resonant feature is robust against fluctuations in the probe position and therefore that the two-probe setup indeed can be a useful characterization tool for the electrical properties of individual nanstructures.

Finally we compare the single and dual probe spectroscopy of the zigzag antidot. Figure 7(d) shows the single-probe spectroscopy both without disorder and including a weak disorder. The single-probe position is the same as one of the probes in Fig. 7(a). We notice small oscillations due to the symmetry breaking caused by the presence of the antidot. This is the same kind of Friedel oscillation arising around single vacancies [15]. Without disorder the localized state is barely visible in Fig. 7(d), but the small resonance vanishes at a disorder strength where it is clearly visible in the dual-probe spectroscopy [cf. Fig. 7(b)]. Consequently the transmission signal from the dual-probe setup yields considerably more information about defect-induced transport processes compared to the single-probe measurement.

\section{CONCLUSION}

A dual-probe setup with probe separation distances in the nanometer range makes it possible to obtain local transport properties on the nanoscale. We have presented a theoretical treatment of such a setup based on a real-space Green's function method. This allows calculation of the transmission between two point probes on an infinite graphene sheet, without requiring periodicity of either probe or sample, while keeping the computational size proportional to the number of modified sites, as opposed to proportional to the total system size.

Directional transport effects, not directly attainable using macroscopic contacts, are explored together with the spectroscopic fingerprints of local perturbations such as vacancies and adatoms. Additionally we show the capability of the dual-probe system to characterize nanostructures. In particular, we observe Fano-type resonances arising from resonant states in adatoms or near edges with a zigzag geometry. The resonance is shown to be a dominant feature in the dual-probe spectroscopy compared to the single probe.

The demonstrated features of the dual-probe setup, such as conductance mapping [41] and spectroscopic analysis, suggest that it has a high potential for applications in the exploration of transport properties on the nanometer scale.

\section{ACKNOWLEDGMENT}

The Center for Nanostructured Graphene (CNG) is sponsored by the Danish Research Foundation, Project No. DNRF58.

\section{APPENDIX: COEFFICIENTS FOR THE SPA}

Below are given the coefficients for the SPA expressions Eq. (6) as derived in [44]:

$$
\begin{aligned}
\mathcal{Q}(E) & = \pm \cos ^{-1}\left(-\sqrt{1-\frac{\mathrm{E}^{2}}{\mathrm{t}^{2}}}\right) \\
\mathcal{A}(E) & =-\frac{1+i}{\sqrt{\pi}} \frac{\sqrt{E}}{\sqrt{\left(E^{2}+3 t^{2}\right) \sqrt{t^{2}-E^{2}}}}, \\
\mathcal{Q}^{+}(E) & = \pm \cos ^{-1}\left(\frac{-t-E}{2 t}\right), \\
\mathcal{Q}^{-}(E) & = \pm \cos ^{-1}\left(\frac{-t+E}{2 t}\right), \\
\mathcal{A}^{+}(E) & =-\frac{1+1 i}{\sqrt{4 \pi}} \sqrt{\frac{E}{|t|(t+E)} \frac{1}{[(3 t+E)(t-E)]^{1 / 4}}}, \\
\mathcal{A}^{-}(E) & =-\frac{1+1 i}{\sqrt{4 \pi}} \sqrt{\frac{E}{|t|(t-E)}} \frac{1}{[(3 t-E)(t+E)]^{1 / 4}} .
\end{aligned}
$$


[1] K. S. Novoselov, D. Jiang, F. Schedin, T. J. Booth, V. V. Khotkevich, S. V. Morozov, and A. K. Geim, Proc. Natl. Acad. Sci. USA 102, 10451 (2005).

[2] C. R. Dean, A. F. Young, I. Meric, C. Lee, L. Wang, S. Sorgenfrei, K. Watanabe, T. Taniguchi, P. Kim, K. L. Shepard, and J. Hone, Nat. Nanotechnol. 5, 722 (2010).

[3] Q. H. Wang, K. Kalantar-Zadeh, A. Kis, J. N. Coleman, and M. S. Strano, Nat. Nanotechnol. 7, 699 (2012).

[4] A. H. Castro Neto, N. M. R. Peres, K. S. Novoselov, and A. K. Geim, Rev. Mod. Phys. 81, 109 (2009).

[5] N. M. R. Peres, F. Guinea, and A. H. Castro Neto, Phys. Rev. B 73, 125411 (2006).

[6] J.-H. Chen, C. Jang, S. Xiao, M. Ishigami, and M. S. Fuhrer, Nat. Nanotechnol. 3, 206 (2008).

[7] G. Binnig, H. Rohrer, C. Gerber, and E. Weibel, Phys. Rev. Lett. 49, 57 (1982).

[8] A. Deshpande and B. J. LeRoy, Physica E 44, 743 (2012).

[9] V. V. Cheianov and V. I. Fal'ko, Phys. Rev. Lett. 97, 226801 (2006).

[10] C. Bena, Phys. Rev. Lett. 100, 076601 (2008).

[11] F. M. D. Pellegrino, G. G. N. Angilella, and R. Pucci, Phys. Rev. B 80, 094203 (2009).

[12] N. M. R. Peres, L. Yang, and S.-W. Tsai, New J. Phys. 11, 095007 (2009).

[13] G. I. Márk, P. Vancsó, C. Hwang, P. Lambin, and L. P. Biró, Phys. Rev. B 85, 125443 (2012).

[14] A. Bergvall and T. Löfwander, Phys. Rev. B 87, 205431 (2013).

[15] J. A. Lawlor, S. R. Power, and M. S. Ferreira, Phys. Rev. B 88, 205416 (2013).

[16] S. Lounis, arXiv:1404.0961.

[17] G. M. Rutter, J. N. Crain, N. P. Guisinger, T. Li, P. N. First, and J. A. Stroscio, Science 317, 219 (2007).

[18] P. Mallet, F. Varchon, C. Naud, L. Magaud, C. Berger, and J.-Y. Veuillen, Phys. Rev. B 76, 041403 (2007).

[19] H. Yang, A. J. Mayne, M. Boucherit, G. Comtet, G. Dujardin, and Y. Kuk, Nano Lett. 10, 943 (2010).

[20] L. Tapaszto, P. Nemes-Incze, G. Dobrik, K. Jae Yoo, C. Hwang, and L. P. Biro, Appl. Phys. Lett. 100, 053114 (2012).

[21] J. Xue, J. Sanchez-Yamagishi, K. Watanabe, T. Taniguchi, P. Jarillo-Herrero, and B. J. LeRoy, Phys. Rev. Lett. 108, 016801 (2012).

[22] J. C. Koepke, J. D. Wood, D. Estrada, Z.-Y. Ong, K. T. He, E. Pop, and J. W. Lyding, ACS Nano. 7, 75 (2013).

[23] S. Hasegawa, I. Shiraki, T. Tanikawa, C. L. Petersen, T. M. Hansen, P. Bøggild, and F. Grey, J. Phys.: Condens. Matter 14, 8379 (2002).

[24] O. Kubo, Y. Shingaya, M. Nakaya, M. Aono, and T. Nakayama, Appl. Phys. Lett. 88, 254101 (2006).

[25] P. Jaschinsky, P. Coenen, G. Pirug, and B. Voigtlander, Rev. Sci. Instrum. 77, 093701 (2006).

[26] T.-H. Kim, Z. Wang, J. F. Wendelken, H. H. Weitering, W. Li, and A.-P. Li, Rev. Sci. Instrum. 78, 123701 (2007).

[27] J. Baringhaus, F. Edler, C. Neumann, C. Stampfer, S. Forti, U. Starke, and C. Tegenkamp, Appl. Phys. Lett. 103, 111604 (2013).

[28] A. Roychowdhury, M. A. Gubrud, R. Dana, J. R. Anderson, C. J. Lobb, F. C. Wellstood, and M. Dreyer, arXiv:1311.1855.

[29] T. Nakayama, O. Kubo, Y. Shingaya, S. Higuchi, T. Hasegawa, C.-S. Jiang, T. Okuda, Y. Kuwahara, K. Takami, and M. Aono, Adv. Mater. 24, 1675 (2012).
[30] A.-P. Li, K. W. Clark, X.-G. Zhang, and A. P. Baddorf, Adv. Funct. Mater. 23, 2509 (2013).

[31] J. Baringhaus, M. Ruan, F. Edler, A. Tejeda, M. Sicot, A.-P. Li, Z. Jiang, E. H. Conrad, C. Berger, C. Tegenkamp, and W. A. de Heer, Nature (London) 506, 349 (2014).

[32] T. Kanagawa, R. Hobara, I. Matsuda, T. Tanikawa, A. Natori, and S. Hasegawa, Phys. Rev. Lett. 91, 036805 (2003).

[33] V. Cherepanov, E. Zubkov, H. Junker, S. Korte, M. Blab, P. Coenen, and B. Voigtländer, Rev. Sci. Instrum. 83, 033707 (2012).

[34] H. Watanabe, C. Manabe, T. Shigematsu, and M. Shimizu, Appl. Phys. Lett. 78, 2928 (2001).

[35] K. W. Clark, X.-G. Zhang, I. V. Vlassiouk, G. He, R. M. Feenstra, and A.-P. Li, ACS Nano 7, 7956 (2013).

[36] K. W. Clark, X.-G. Zhang, G. Gu, J. Park, G. He, R. M. Feenstra, and A.-P. Li, Phys. Rev. X 4, 011021 (2014).

[37] T.-H. Kim, X.-G. Zhang, D. M. Nicholson, B. M. Evans, N. S. Kulkarni, B. Radhakrishnan, E. A. Kenik, and A.-P. Li, Nano Lett. 10, 3096 (2010).

[38] F. R. Eder, J. Kotakoski, K. Holzweber, C. Mangler, V. Skakalova, and J. C. Meyer, Nano Lett. 13, 1934 (2013).

[39] S.-H. Ji, J. B. Hannon, R. M. Tromp, V. Perebeinos, J. Tersoff, and F. M. Ross, Nat. Mater. 11, 114 (2012).

[40] P. W. Sutter, J.-I. Flege, and E. A. Sutter, Nat. Mater. 7, 406 (2008).

[41] M. Settnes, S. R. Power, D. H. Petersen, and A.-P. Jauho, Phys. Rev. Lett. 112, 096801 (2014).

[42] S. Datta, Electronic Transport in Mesoscopic Systems (Cambridge University Press, Cambridge, UK, 1997).

[43] S. Reich, J. Maultzsch, C. Thomsen, and P. Ordejón, Phys. Rev. B 66, 035412 (2002).

[44] S. R. Power and M. S. Ferreira, Phys. Rev. B 83, 155432 (2011).

[45] A. Lherbier, S. M. M. Dubois, X. Declerck, Y.-M. Niquet, S. Roche, and J.-C. Charlier, Phys. Rev. B 86, 075402 (2012).

[46] L. E. F. Foa Torres, S. Roche, and J.-C. Charlier, Introduction to Graphene-Based Nanomaterials (Cambridge University Press, Cambridge, UK, 2014).

[47] H. Haug and A.-P. Jauho, Quantum Kinetics in Transport and Optics of Semiconductors (Springer, Berlin, Heidelberg, 2008).

[48] E. N. Economou, Green's Functions in Quantum Physics (Springer, Berlin, Heidelberg, 2005).

[49] J. Tersoff and D. R. Hamann, Phys. Rev. Lett. 50, 1998 (1983).

[50] V. Meunier and P. Lambin, Phys. Rev. Lett. 81, 5588 (1998).

[51] H. Amara, S. Latil, V. Meunier, P. Lambin, and J.-C. Charlier, Phys. Rev. B 76, 115423 (2007).

[52] Y. Zhang, V. W. Brar, F. Wang, C. Girit, Y. Yayon, M. Panlasigui, A. Zettl, and M. F. Crommie, Nat. Phys. 4, 627 (2008).

[53] A. Fasolino, J. H. Los, and M. I. Katsnelson, Nat. Mater. 6, 858 (2007).

[54] J. P. Robinson, H. Schomerus, L. Oroszlány, and V. I. Fal'ko, Phys. Rev. Lett. 101, 196803 (2008).

[55] T. O. Wehling, S. Yuan, A. I. Lichtenstein, A. K. Geim, and M. I. Katsnelson, Phys. Rev. Lett. 105, 056802 (2010).

[56] B. Uchoa, L. Yang, S. W. Tsai, N. M. R. Peres, and A. H. Castro Neto, Phys. Rev. Lett. 103, 206804 (2009).

[57] U. Fano, Phys. Rev. 124, 1866 (1961). 
[58] F. Schedin, A. K. Geim, S. V. Morozov, E. W. Hill, P. Blake, M. I. Katsnelson, and K. S. Novoselov, Nat. Mater. 6, 652 (2007).

[59] A. Cagliani, D. Mackenzie, L. Tschammer, F. Pizzocchero, K. Almdal, and P. Bøggild, Nano Res. 7, 743 (2014).

[60] J. A. Fürst, J. G. Pedersen, C. Flindt, N. A. Mortensen, M. Brandbyge, T. G. Pedersen, and A. P. Jauho, New J. Phys. 11, 095020 (2009).
[61] T. G. Pedersen, C. Flindt, J. G. Pedersen, N. A. Mortensen, A.-P. Jauho, and K. Pedersen, Phys. Rev. Lett. 100, 136804 (2008).

[62] J. G. Pedersen, T. Gunst, T. Markussen, and T. G. Pedersen, Phys. Rev. B 86, 245410 (2012).

[63] T. Gunst, T. Markussen, A.-P. Jauho, and M. Brandbyge, Phys. Rev. B 84, 155449 (2011). 